\title{
Envasado de Carne de Vacuno con Hueso y Grasa en Atmósfera Modificada con $\mathrm{CO}_{2}$ y $\mathrm{CO}$
}

\author{
Estrella R. Aspé ${ }^{1}$, Marlene D. Roeckel ${ }^{1}$, Cristina Martí ${ }^{2}$ y Romel Jiménez ${ }^{1}$ \\ Universidad de Concepción, (1) Departamento de Ingeniería Química, (2) Departamento de \\ Farmacología, Correo 3, Casilla 160-C, Concepción-Chile (e-mail: easpe@udec.cl)
}

\begin{abstract}
Resumen
Se evaluó el efecto de envasado en atmósfera modificada (MAP) con bajo contenido de CO $(0.1 \%$ y $0.4 \%$ ) y presencia de $\mathrm{CO}_{2}$ sobre el color rojo y la vida útil de carne de vacuno. Se determinaron características microbiológicas, fisicoquímicas, organolépticas-sensoriales y de color del músculo y se compararon los resultados con el MAP de $70 \% \mathrm{O}_{2}+30 \% \mathrm{CO}_{2}$. La vida útil de las muestras con $\mathrm{O}_{2}$ fue de 30 días mientras que para las muestras en MAP la vida útil fue de 40 a 45 días y no dependió de la concentración de CO. La intensidad del color rojo para las muestras con CO aumentó en forma sostenida en el tiempo, mientras que las envasadas con $\mathrm{O}_{2}$ presentaron un máximo al día 16. Para $0.4 \%$ de $\mathrm{CO}$ la intensidad del color rojo se mantuvo durante nueve semanas y la presencia de $\mathrm{CO}$ no afectó al crecimiento microbiano. La rancidez fue mayor en la carne con $\mathrm{O}_{2}$.
\end{abstract}

Palabras clave: carne de vacuno, envasado de carne, atmósfera modificada, vida útil

\section{Packaging of Bone and Fat Containing Beef Meat under a Modified Atmosphere Containing $\mathrm{CO}_{2}$ and $\mathrm{CO}$}

\begin{abstract}
The effects of modified atmosphere packaging (MAP) containing $\mathrm{CO}_{2}$ and low $\mathrm{CO}$ concentrations $(0.1$ and $0.4 \%)$ on the red color of beef meat and on its shelf life were evaluated. Microbiological, physico-chemical and organoleptic-sensorial characteristics and color of the meat were determined and the results were compared to those of meat packed under $70 \% \mathrm{O}_{2}+30 \% \mathrm{CO}_{2}$ atmosphere. The shelf life of meat packed under $\mathrm{O}_{2}$ was 30 days while for the MAP-packed samples the shelf life was 40-45 days and did not vary with the $\mathrm{CO}$ concentration used. The intensity of the red color of the meat packed with $\mathrm{CO}$ steadily increased with time, while samples packed under $\mathrm{O}_{2}$ reached a peak at day 16 . The red color intensity remained unchanged for nine weeks in samples packed with $0.4 \%$ $\mathrm{CO}$ and the presence of $\mathrm{CO}$ did not affect the bacterial growth rate. Rancidity was higher in meat packed with $\mathrm{O}_{2}$ atmosphere.
\end{abstract}

Keywords: beef meat, meat packaging, modified atmosphere, shelf life 


\section{INTRODUCCIÓN}

Las exigencias actuales de vida útil de productos cárnicos debe exceder las 5 semanas; entendiéndose por vida útil el máximo tiempo de almacenamiento antes de que la carne pierda su calidad nutricional, sensorial y de seguridad alimenticia al nivel de ser rechazada por los consumidores (Masana et al., 2006). En este sentido, las características microbiológicas y organolépticas del producto son determinantes, donde el color de la carne juega un papel fundamental en la relación calidad-apariencia que el consumidor establece al elegir uno u otro producto (Farber y Dodds, 1995). Así, resulta imprescindible la continua búsqueda de sistemas de envasado de carne innovadores, con el objetivo de colocar un producto cárnico en un mercado ya consolidado.

En la actualidad, una de las alternativas de envasado de carne destinada al mercado minorista es el Envasado en Atmósfera Modificada (MAP, por las siglas en inglés de Modified Atmosphere Packaging), que, términos generales, consiste en envasar alimentos en una atmósfera con composición distinta a la del aire (Farber y Dodds, 1995). De acuerdo con esta definición, varios sistemas de envasado se clasifican como MAP, dentro de los cuales, los más utilizado son el envasado al vacío, con vida útil superior a 70 días (Nissen et al., 1996) y el envasado en atmósfera con alta concentración de $\mathrm{O}_{2}$ en $\mathrm{CO}_{2}$. El primero con el inconveniente de generar un color café oscuro en la carne, de baja aceptación por el consumidor; en el segundo sistema de envasado, si bien mantiene el color de la carne fresca, ésta tiene una limitada vida útil (10 días a $4^{\circ} \mathrm{C}$ ) debido a la alta concentración de oxígeno y el consiguiente desarrollo de la flora aeróbica (Sørheim et al., 1999; Jayasingh et al., 2001).

Otro efecto sobre la calidad de la carne, relacionado con el uso de atmósferas con alto contenido de $\mathrm{O}_{2}$, es la oxidación de lípidos, que se asocia al desarrollo de malos olores y sabores, los que generalmente se denominan como rancios. Ese proceso indeseado se inicia a nivel de membrana celular, con un mecanismo autocatalítico en cadena con radicales libres. Se ha encontrado una buena correlación entre la aceptación de la carne por el consumidor y niveles límites de rancidez, expresada a través del consumo de ácido tiobarbitúrico (TBARS) en caracterizaciones fisicoquímicas. Sin embargo, en el mercado nacional el sistema más utilizado en MAP de carne de vacuno consiste en una mezcla con $70 \%$ de $\mathrm{O}_{2}$ y un $30 \%$ de $\mathrm{CO}_{2}$. De acuerdo a información empírica obtenida en la industria, la carne envasada en esta atmósfera tiene una vida útil en el mercado del "retail" de, aproximadamente 12 días.

Respecto al color rojo de la carne, éste depende fundamentalmente del estado de oxidación de la mioglobina (MB), la cual representa el 80 a $90 \%$ de los pigmentos de la carne (SCF, 2001). La mioglobina es una proteína globular que posee un centro activo protoheme (ferrous protoprophyrin IX), que es responsable del enlace $\mathrm{O}_{2}-\mathrm{MB}$ (Austin et al., 1974), y su función es almacenar y facilitar la difusión del oxígeno desde los capilares a la mitocondria (Austin et al., 1974); por ello es capaz de asociarse y disociarse rápidamente con la molécula de $\mathrm{O}_{2}$, en función de la presión parcial a la que esté expuesta la carne (Farber y Dodds, 1995). Esta molécula también posee gran afinidad por otras moléculas biatómicas, como el $\mathrm{CO}$, debido a su forma y polaridad (Blomberg et al., 2005). En la figura 1 se esquematizan los distintos estados de oxidación de esta molécula en función del ligando al que se encuentra unida.

Una alternativa para mejorar tanto la calidad organoléptica como la vida útil microbiológica de la carne es el uso del CO en atmósferas modificadas (Sørheim et al., 1997; Otwell et al., 2006). El uso de este gas en mezclas con $\mathrm{CO}_{2}$ y ausencia de $\mathrm{O}_{2}$ en MAP, ha sido aceptado por la FDA U.S. y ha demostrado reducir el deterioro por oxidación de lípidos y proteínas, retardar el desarrollo de los microorganismos responsables de la descomposición de la carne y generar un color más estable en el producto (SCF, 2001).

A pesar de las suficientes referencias disponibles que evalúan los efectos del envasado de carne en atmósferas modificadas, no existen experiencias citadas en la literatura donde se desarrollen ensayos a nivel piloto e industrial con cortes de vacuno con hueso, y grasa, como es el caso de los resultados que se presentan y discuten en este trabajo. 


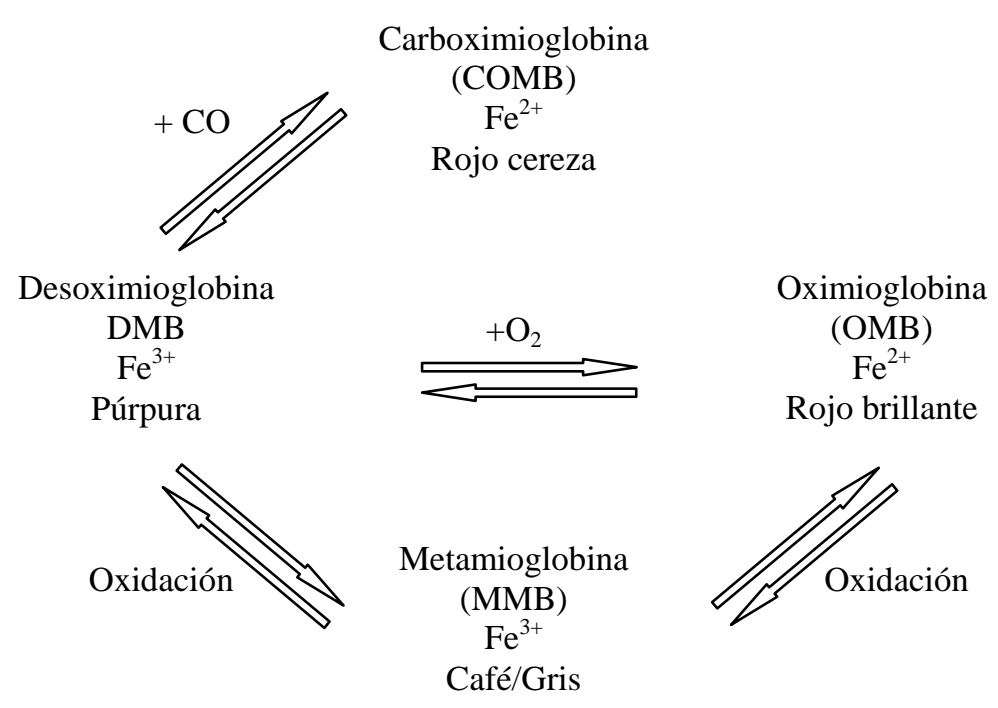

Fig. 1: Esquema que representa el color de la carne en función de los estados de oxidación de la mioglobina.

\begin{abstract}
A partir de los antecedentes expuestos, el objetivo de este trabajo fue experimentar, a escala piloto e industrial, nuevas alternativas de envasado de carnes de vacuno destinada al mercado nacional, en Chile. Para ello, se realizaron estudios del efecto de uso de atmósferas con bajo nivel de $\mathrm{CO}$ en $\mathrm{CO}_{2}$ y con alta concentración de $\mathrm{O}_{2}$ en $\mathrm{CO}_{2}$ sobre la carne de vacuno con contenido graso y hueso. Este estudio se realizó mediante el seguimiento de propiedades microbiológicas, fisicoquímicas y organolépticas de la carne, en función del tiempo de almacenamiento, determinándose la vida útil microbiológica del producto.
\end{abstract}

\title{
MATERIALES Y MÉTODOS
}

Se utilizaron 458 chuletones de vacuno de lomo liso (Longissimus dorsi con hueso y grasa), con un peso entre 400 y $500 \mathrm{~g}$, y largo y ancho promedio de 16.2 y $6.8 \mathrm{~cm}$, respectivamente. Los cortes utilizados fueron extraídos de animales de la misma raza (Clavel alemán) y criados bajo condiciones similares de alimentación. La materia prima se caracterizó mediante análisis fisicoquímicos, microbiológicos y organolépticos en duplicado. Para este fin, se usaran ocho muestras.

Se utilizó una atmósfera de envasado con $70 \% \mathrm{O}_{2} / 30 \% \mathrm{CO}_{2}$, que fue denominada Atmósfera 0 , y que se utilizó como blanco. Esta mezcla es la utilizada por la industria nacional para los productos en MAP. Además, se ensayaron dos atmósferas con diferentes concentraciones de $\mathrm{CO}$ en $\mathrm{CO}_{2}$ y $\mathrm{N}_{2}$ : la primera compuesta por $0.1 \% \mathrm{CO} / 20 \% \mathrm{CO}_{2} / 79.9 \% \mathrm{~N}_{2}$ (Atmósfera 1) y la segunda por $0.4 \% \mathrm{CO} /$ $20 \% \mathrm{CO}_{2} / 79.6 \% \mathrm{~N}_{2}$ (Atmósfera 2). Las concentraciones de $\mathrm{CO}$ utilizadas consideran la normativa impuesta por la FDA (2001), y responden a estudios previos (Sørheim et al., 1997), donde se demostró que para concentraciones menores al $1 \%$ de $\mathrm{CO}$ en las atmósferas de envasado, se generan mejoras significativas en el color de la carne envasada en atmósfera anaerobia. Todos los gases utilizados fueron proporcionados por INDURA y sus concentraciones fueron verificadas mediante análisis cromatográfico en el Laboratorio de Carbones del Departamento de Ingeniería Química de la Universidad de Concepción.

Se utilizaron bolsas tipo pouch T7330B de $30 \times 40 \mathrm{~cm}$, proporcionadas por Cryovac, con un espesor de $76 \mu \mathrm{m}$, velocidad de transferencia de oxígeno (OTR) de $4 \mathrm{~cm} 3 /(\mathrm{m} 2$ día), y una velocidad de transmisión de vapor húmedo (MVTR) de $7.7 \mathrm{~g} / \mathrm{m} 2 /$ día. Sus dimensiones fueron determinadas en función del volumen del producto dispuesto en la bandeja $(2 \mathrm{~L})$ y del largo necesario para la operación en la máquina de envasado $(40 \mathrm{~cm})$, con lo que resulta una bolsa de $4 \mathrm{~L}$ de capacidad. También se determinó que el porcentaje de reducción del volumen debido a la disolución de los gases en la atmósfera es de un $6 \%$, en el caso de la atmósfera con alto nivel de $\mathrm{O}_{2}$, y de un $4 \%$ 
para cualquiera de las atmósferas con bajo nivel de CO. Así, en ambos casos, el volumen de gas contenido es siempre mayor a $3 \mathrm{~L}$, con lo que se evita el colapso de la bolsa. La razón gas/producto en los tres tipos de atmósferas osciló entre 6 y $7.5 \mathrm{~L} / \mathrm{kg}$. Para evitar la ruptura de las bolsas por el hueso del chuletón, se utilizaron bandejas de poliestireno espumado marca Linpac 4S, de 26,5 x 18.9 x $1.5 \mathrm{~cm}$; para evitar el aparente exudado se usaron almohadillas absorbentes de la misma marca.

El proceso de envasado en atmósfera modificada se realizó en una máquina Multivac, C 200, proporcionada por Indura, con un ciclo de envasado MAP en 4 etapas: 1) evacuación del aire, a 40 mbar de presión, 2) alimentación de gas, a una presión de $750 \mathrm{mbar}$, 3) sellado de bolsa durante 4 segundos, 4) nivelación de la presión interna de la cámara hasta la atmosférica.

Cada chuletón fue colocado en una bandeja sobre una almohadilla absorbente y depositado dentro de una bolsa pouch, para posterior almacenamiento en un túnel de frío a una temperatura de $-30^{\circ} \mathrm{C}$ durante $1 \mathrm{~h}$. El objetivo de esta etapa de sobreenfriamiento es aumentar la disolución de los gases (CO y CO2) en la carne. En esta etapa, los chuletones fueron dispuestos en cajas de plástico abiertas (20 chuletones/bandeja), y luego apilados en "pallet" para ser depositados en el túnel de frío. Se definió 1 hora como el tiempo necesario de permanencia de los chuletones en el túnel de frío. Este es el tiempo en el cual se congela el $50 \%$ del agua en la carne. Los chuletones fueron trasladados a la zona de envasado a una temperatura promedio de $7^{\circ} \mathrm{C}$, donde fueron distribuidos de forma aleatoria en tres grupos. Los dos primeros, de 200 chuletones cada uno, fueron envasados en Atmósfera 1 y Atmósfera 2, y los 50 restantes con Atmósfera 0. ( chuletones fueron utilizados para la caracterización de la materia prima. Todas las cámaras de frío donde se realizó el almacenamiento de las muestras se mantuvieron oscuras.

Se realizaron recuentos bacterianos aerobios mesófilos y anaerobios mesófilos, así como recuento de lactobacilos, de acuerdo con la metodología señalada en el Bacteriological Analytical Manual, BAM (FDA, 2001).

Para establecer el tiempo de vida útil del producto en relación a su calidad microbiológica, se adoptó el criterio establecido en "EL REGLAMENTO SANITARIO DE LOS ALIMENTOS DTO. № 977/96" del Ministerio de Salud, Chile, donde se especifica que el límite para el Recuento Aerobio Mesófilo, para el cual el alimento no representa riesgo para la salud es de $10^{6}(\mathrm{UFC} / \mathrm{g})$, considerándose riesgoso cuando ha excedido $10^{7}$ (UFC/g).

\section{Caracterización físico-química}

El exudado se determinó a través de la diferencia entre los pesos de la muestra húmeda envasada y la muestra y envases secos; para ello se utilizó una balanza con $0.1 \mathrm{~g}$ de precisión.

La humedad de las muestras se determinó en estufa a $105^{\circ} \mathrm{C}$, hasta obtener peso constante en una balanza de $0.1 \mathrm{~g}$ de precisión.

Para el $\mathrm{pH}$, se añadió $90 \mathrm{~mL}$ de agua destilada a $10 \mathrm{~g}$ de carne molida y se homogeneizó con ultraturrex (Ultraturrex Karl Corp.) durante $1 \mathrm{~min}$. Se filtró el homogeneizado y se estandarizó el medidor de $\mathrm{pH}$ ( $\mathrm{pH}$ meter 240 Corning) con solución tampón de $\mathrm{pH} 4.0$ y 7.0 . Se midió el pH al filtrado.

Para determinar la retención de agua en las muestras, se mezclaron $4 \mathrm{~mL}$ de solución de $\mathrm{NaCl} 0.6 \mathrm{M}$ con $2 \mathrm{~g}$ de carne molida y se colocaron en un tubo de centrífuga. Se agitó con varilla de vidrio por 1 min y se colocó en baño de hielo por $30 \mathrm{~min}$. Se agitó nuevamente por 1 min y se centrifugó a 8000 rpm durante $15 \mathrm{~min}$. El sobrenadante fue decantado y medido su volumen. Se reporta el volumen de $\mathrm{NaCl} 0.6 \mathrm{M}$ retenido por $\mathrm{kg}$ de músculo.

La determinación de la rancidez, se basó en el grado de oxidación de lípidos en la carne. Se determinó según procedimiento utilizado por Pensel en su tesis de Master (Pensel, 1990). Se adicionaron $12.5 \mathrm{~mL}$ de TCA 20\% (ácido tricloroacético preparado en ácido metafosfórico 1.6\%) a $5 \mathrm{~g}$ de carne molida y se homogenizó durante $2 \mathrm{~min}$. Se agregaron $12.5 \mathrm{~mL}$ de agua y se homogenizó 
durante 30s. La mezcla se filtró y recogió en una probeta. Por otra parte, se preparó una solución de TBA (ácido tiobarbitúrico) $0.02 \mathrm{M}$ y se mezclaron $5 \mathrm{~mL}$ de solución de TBA con $5 \mathrm{~mL}$ de solución filtrada. Para el "blanco" se sustituyó la solución de TBA por agua destilada. Las muestras se incubaron a $70^{\circ} \mathrm{C}$ por 1 hora. Se midió la absorbancia de la solución en espectrofotómetro (Spectronic 20 Genesys) a $530 \mathrm{~nm}$ y en base a una curva de calibración, previamente elaborada, se determinó la concentración de malonaldehido por kilogramo de muestra (mg de malonaldehido / $\mathrm{kg}$ de carne), que se asocia al grado de oxidación de lípidos en la carne.

Se desarrolló la evaluación sensorial de carne cruda y cocida, a través de un panel de 10 jueces entrenados (panel de 10 jueces). En esta evaluación se evaluó la calidad de la carne usando una escala descriptiva hedónica (1-7), de acuerdo a parámetros predefinidos. Primeramente se evaluó el color y olor en carne cruda, por el panel sensorial. Para esta evaluación transcurrió un tiempo aproximado de 1 hora, desde la apertura de las bolsas hasta completar la evaluación por todos los jueces. Adicionalmente se realizó la evaluación sensorial de olor, sabor, jugosidad y textura en carne cocida. Las muestras cocidas fueron preparadas según el procedimiento establecido por AMSA (American Meat Science Association-AMSA, 1995), que consiste en llevar a cabo el grillado de la carne en un Burger Grill cerrado, mediante un calentamiento paulatino hasta lograr $71^{\circ} \mathrm{C}$ en el centro de la carne. La temperatura de la carne fue medida constantemente con un termómetro de punción.

El análisis organoléptico fue desarrollado por la presidenta del Panel Sensorial (un juez) y consiste en calificar el color y olor de la carne cruda inmediatamente después que es abierto el envase del producto. Se utilizó una escala de intensidad hedónica de 1 a 7 . El procedimiento utilizado correspondió al establecido por AMSA (1995; 2005).

Se determinaron las coordenadas colorimétricas mediante el uso de un equipo COLORFLEX (Chroma Meter, Minolta, modelo CR-200) que proporciona la Luminosidad $\left(L^{*}\right)$ y la Cromaticidad $\left(a^{*}\right.$, $\left.b^{\star}\right)$. El parámetro $a^{\star}$, que entrega la variación del color entre el rojo y el verde, fue utilizado para comparar el color rojo en las muestras de carne (Hui et al., 2006; Mendez et al., 2006). Se le practican 6 mediciones por cada cara a cada muestra y el tiempo total de medición por muestra no excede los 40 segundos.

Análisis de Composición de gases: Se determinó la fracción molar de $\mathrm{CO}, \mathrm{CO}_{2}, \mathrm{O}_{2}, \mathrm{y} \mathrm{N}_{2}$, en los laboratorios de Indura mediante cromatografía (Perkin Elmer serie 9000), con detector de ionización de llama (TCD) y con helio como gas carrier. El método de muestreo consistió en la inyección septum con jeringas estériles. El método de medición tiene una sensibilidad analítica de $\pm 2 \%$.

Se aplicó un análisis de Varianza de dos vías (Two Way Anova) para el examen de las distintas magnitudes estudiadas. La hipótesis planteada fue que los efectos asociados a los distintos tratamientos (tiempo o composición de atmósfera) sobre la magnitud observada son iguales entre sí e iguales a 0 . Así, bajo la suposición de que los errores son independientes y se distribuyen normalmente con media 0 es posible determinar si los tratamientos estudiados tienen algún efecto sobre el observado. Se utilizó el programa "SPSS 15.0 for Windows Evaluation Version", aplicando un test $F$, que requiere como parámetro el nivel de significancia $\mathrm{p}$ (probabilidad de rechazar la hipótesis planteada, siendo verdadera). Se fijó el valor máximo de $p$ en 0.05 en consideración al error experimental típico.

\section{RESULTADOS Y DISCUSIÓN}

\section{Caracterización Microbiológica}

A partir del día 20 de envasado, el recuento bacteriano mesófilo aerobio (Fig. 2) mostró diferencias significativas (cercana a la incertidumbre experimental) entre la Atmósfera 0 y las Atmósferas 1 y 2 $(p<0.05)$. Esto puede atribuirse a dos factores, que han sido claramente descritos en la literatura especializada. Por una parte, la mayor concentración de $\mathrm{CO}_{2}$ presente en la Atmósfera 0 (30\%) puede extender la fase estacionaria del crecimiento de los microorganismos y generar una retardo en el desarrollo microbiológico, pudiendo disminuir el crecimiento de Brochothrix thermosphacta. (Dixon et al., 1991; Gill, 1996 y Nissen et al., 1996). Un segundo factor que puede explicar estos resultados 
es la alta concentración de oxígeno utilizada en la Atmósfera 0. En este sentido Zang y Sundar (2005), reportaron que para concentraciones de $\mathrm{O}_{2}$ por encima del $35 \%$ se observó una reducción significativa en el crecimiento microbiano para carne de cerdo envasada en atmósfera modificada. Este resultado coincide a su vez con trabajos publicados anteriormente (Brody, 2000).

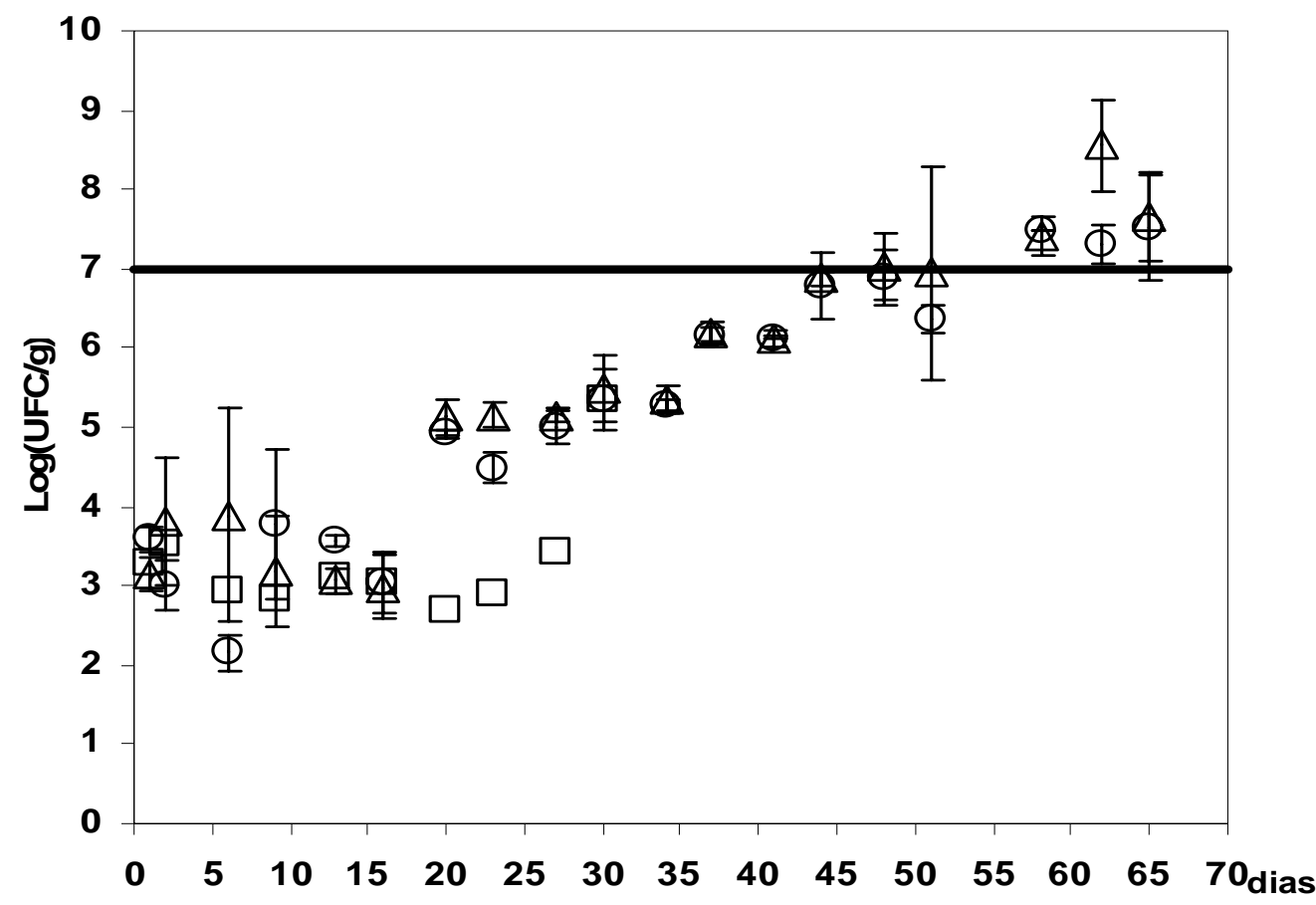

Fig. 2: Recuento bacteriano mesófilo aerobio en carne de vacuno envasada en MAP (valor promedio, barra. $\pm \sigma)$. $\square$ Atmósfera 0, $\bigcirc$ Atmósfera 1, $\triangle$ Atmósfera 2, — Límite admisible por el Reglamento Sanitario para los alimentos.

El recuento mesófilo anaerobio presentó un comportamiento similar al aerobio (no se muestra). Se descartó algún efecto del $\mathrm{CO}$, para las dos concentraciones utilizadas, sobre el desarrollo de los microorganismos a través del análisis estadístico $(p>0.05)$; este resultado concuerda con estudios anteriores, donde se estableció que en mezclas con altos niveles de $\mathrm{CO}_{2}$ se enmascara el efecto inhibitorio de bajas concentraciones de CO (Luño et al., 2000).

\section{Caracterización Físico-Química}

Los resultados de la caracterización fisico-química, obtenidos para los 65 días de ensayo, demuestran que la composición de los gases de envasado no tiene efecto significativo sobre el exudado, el $\mathrm{pH}$ y la humedad en la carne de vacuno envasada en MAP ( $p>0.05)$. Para las tres atmósferas ensayadas se observa un aumento del exudado y del $\mathrm{pH}$ con el tiempo de envasado (no se muestra).

Sin embargo, se observaron valores significativamente menores $(p<0.05)$ en la capacidad de retención de agua (CRA) en las muestras envasadas en Atmósfera 0 en comparación con las muestras envasadas a distintas concentraciones de CO (Fig. 3).

Si bien es cierto, el factor más influyente en la CRA es el estado fisicoquímico de las proteínas, existe evidencia que la oxidación de los lípidos puede afectar la CRA (Brannan y Decker, 2001). Así, el alto contenido de oxígeno en la Atmósfera 0 favorecería la oxidación de los lípidos de la membrana celular, lo que disminuiría la capacidad de retención de agua a nivel intracelular.

También se generaron niveles significativamente mayores de rancidez $(p<0.05)$ en la carne envasada en Atmósfera 0, tanto en el músculo (Fig. 4) como en la grasa de las mismas muestras (Fig. 5); esto sería producto de los altos niveles de oxígeno presentes en esta atmósfera, que 
favorecen la oxidación de lípidos. Esto concuerda con resultados previos (John et al., 2005), donde se compara la oxidación de lípidos en carne envasada en atmósferas con alta y baja concentración de $\mathrm{O}_{2}$.

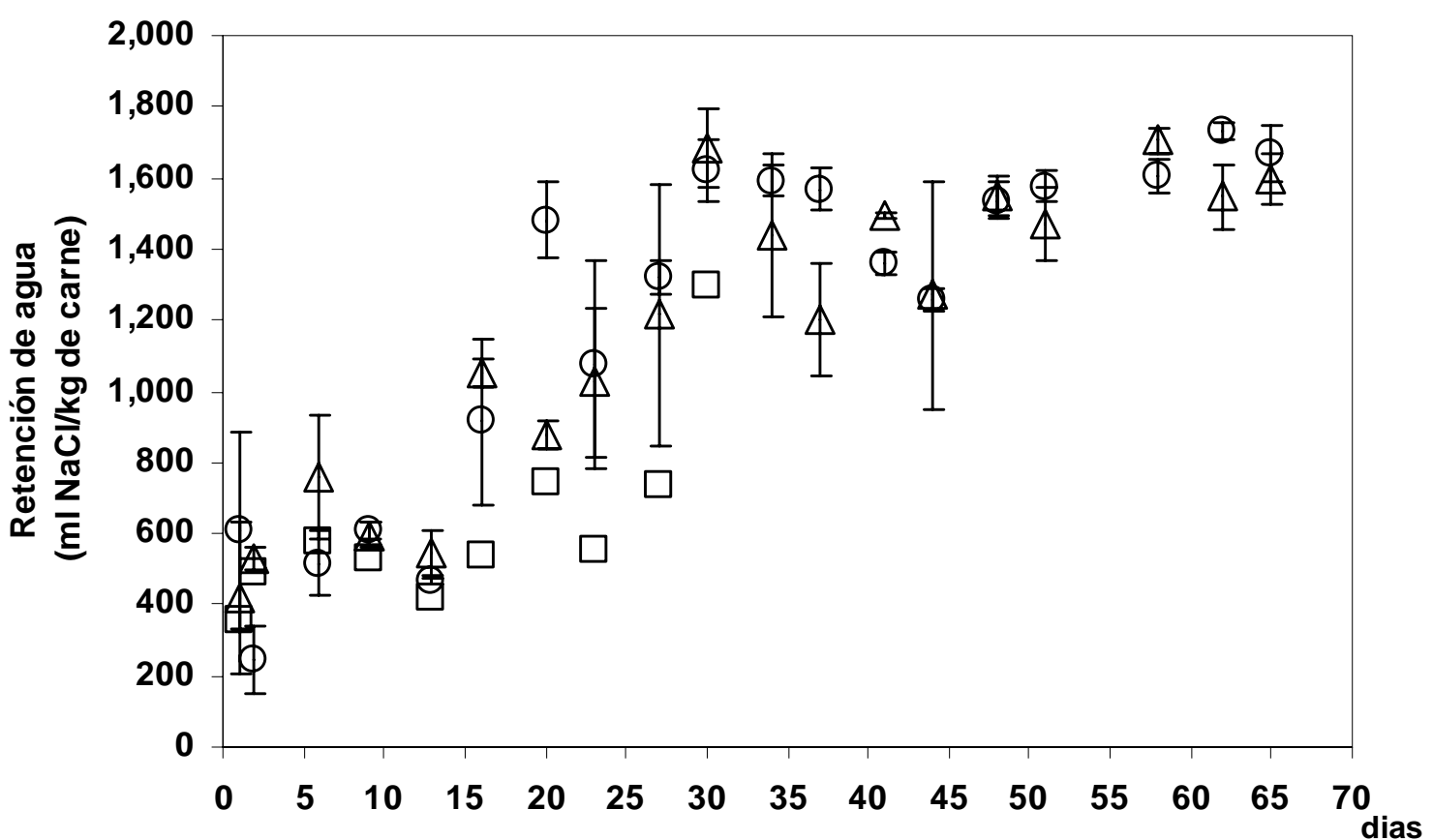

Fig. 3: Capacidad de retención de agua en carne de vacuno envasada en MAP (valor promedio, barra. $\pm \sigma)$. $\square$ Atmósfera 0, $\bigcirc$ Atmósfera 1, $\triangle$ Atmósfera 2.

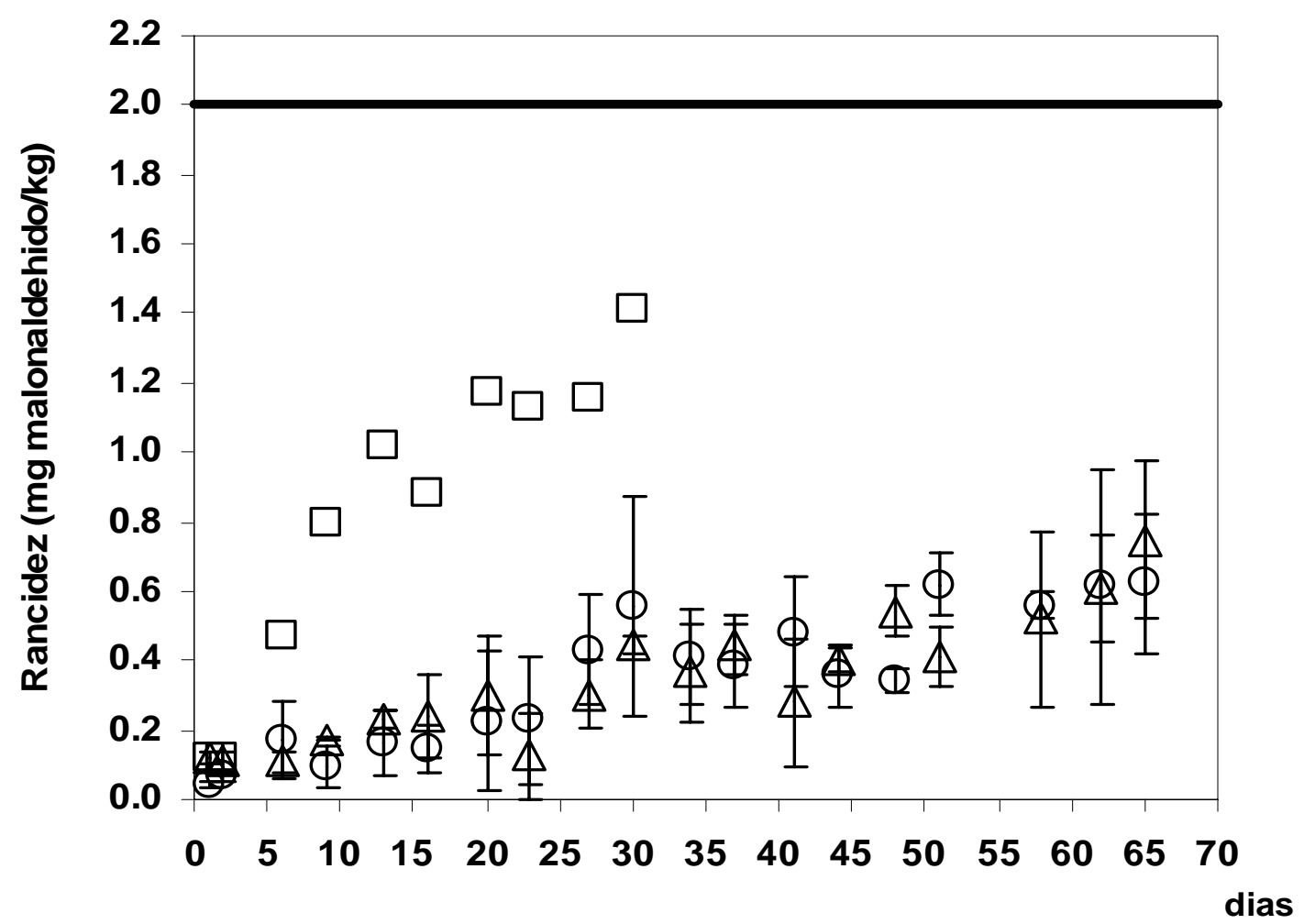

Fig. 4: Rancidez del músculo de carne de vacuno envasada en MAP (valor promedio, barra. $\pm \sigma$ ).

$\square$ Atmósfera 0, O Atmósfera 1, $\triangle$ Atmósfera 2,

(Campo et al., 2006). 
El nivel de compuestos reactivos al ácido tiobarbitúrico (TBARS) detectado en la grasa subcutánea es menor al detectado en el músculo de la carne (Figs. 4 y 5), lo que se debe a que la grasa subcutánea presenta menores niveles de ácidos grasos poliinsaturados, más susceptibles a la oxidación (Campo et al., 2006). No obstante, bajo ninguna de las atmósferas estudiadas se alcanza el valor de $2 \mathrm{mg}$ de TBARS $/ \mathrm{kg}$, considerado como límite de aceptabilidad sensorial en cuanto a la oxidación de lípidos (Chow, 1992).

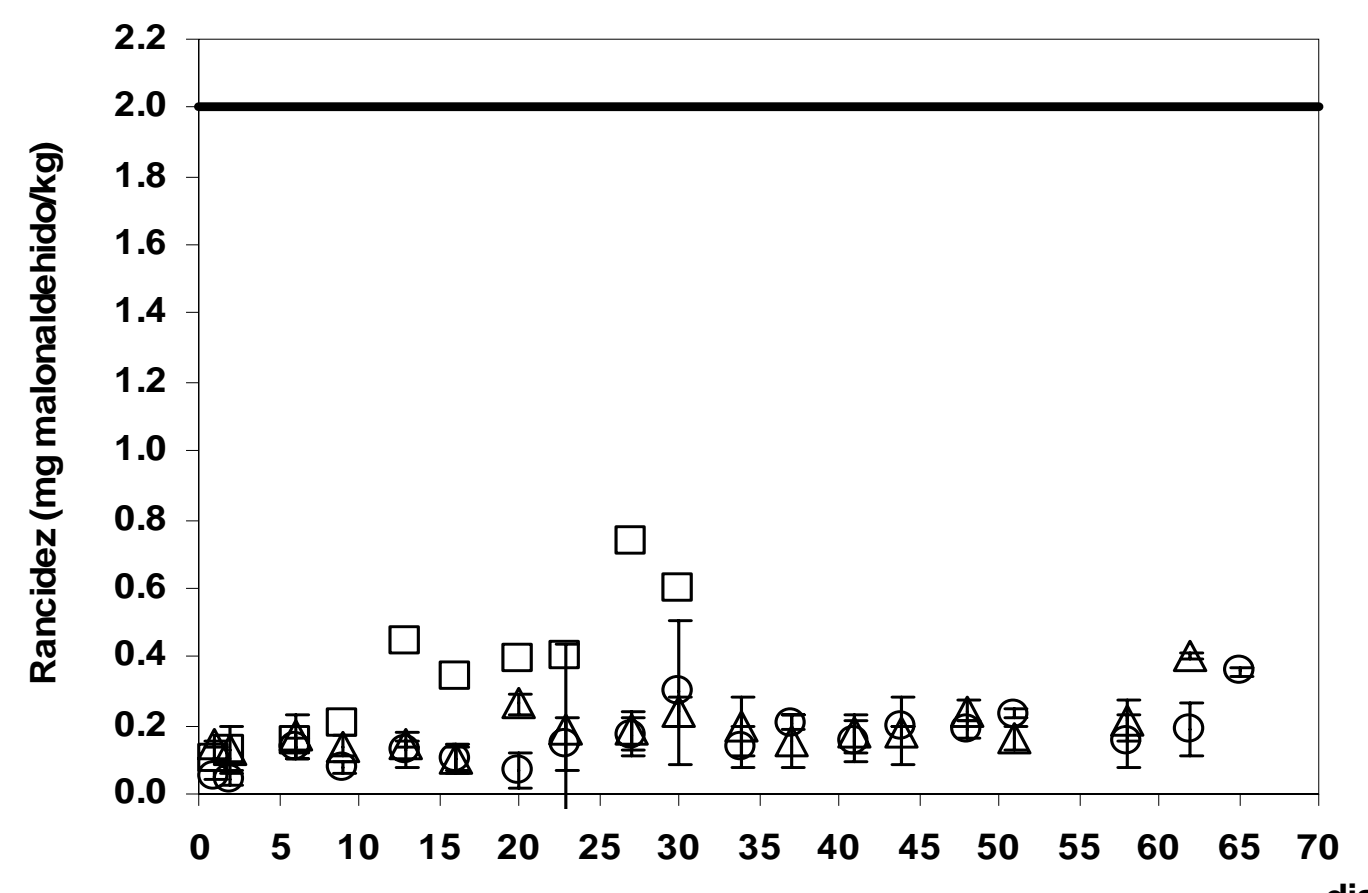

Fig. 5: Rancidez de la grasa en carne de vacuno envasada en MAP (valor promedio, barra. $\pm \sigma$ ).

$\square$ Atmósfera 0, ○ Atmósfera 1, $\triangle$ Atmósfera 2, — Límite de aceptabilidad sensorial

(Campo et al., 2006).

Caracterización organoléptica (por un juez), sensorial (por panel de 10 jueces) y color instrumental.

La evaluación organoléptica y sensorial de color, durante los primeros 30 días de ensayo para carne cruda, no indicó diferencias significativas $(p>0.05)$ entre las muestras envasadas en Atmósfera 0 y Atmósfera 2 (Fig. 6). Sin embargo, las muestras envasadas bajo estas dos atmósferas resultaron significativamente mejor evaluadas en cuanto a olor y color $(p<0.05)$ que las envasada con Atmósfera 1.

Por su parte, la evaluación sensorial de olor para carne cruda indicó evaluaciones significativamente superiores $(p<0.05)$ para las muestras con CO que para las envasadas en Atmósfera 0 (Fig. 7). Sin embargo, estas diferencias no se reflejaron de igual forma en las evaluaciones organolépticas. Este resultado se atribuye a que, durante el tiempo de espera requerido para la evaluación sensorial (aproximadamente de 1 hora a temperatura ambiente), los volátiles producidos en las muestras con $\mathrm{CO}$, entre ellos los derivados del ácido láctico, se liberen. De esta forma, el producto queda relativamente inodoro. Sin embargo, las muestras en Atmósfera 0 mantienen su tendencia con el avance del tiempo de almacenamiento, a pesar del discreto crecimiento microbiano observado para esta atmósfera. Esto sugiere la influencia de otros factores como la rancidez, que resultó superior para este tipo de atmósfera. Por su parte, la evaluación organoléptica, al efectuarse en pocos segundos después de abierta la bolsa, no acusa diferencias significativas de olor entre las tres atmósferas ensayadas.

La evaluación sensorial de olor y textura para carne cocida no mostró diferencias significativas $(p>0.05)$ entre las tres atmósferas MAP comparadas. Sin embargo, sí se observaron diferencias 
significativas en las evaluaciones de sabor y jugosidad $(p<0.05)$, siendo mejor evaluadas las muestras envasadas en Atmósfera 2 por el panel de jueces.
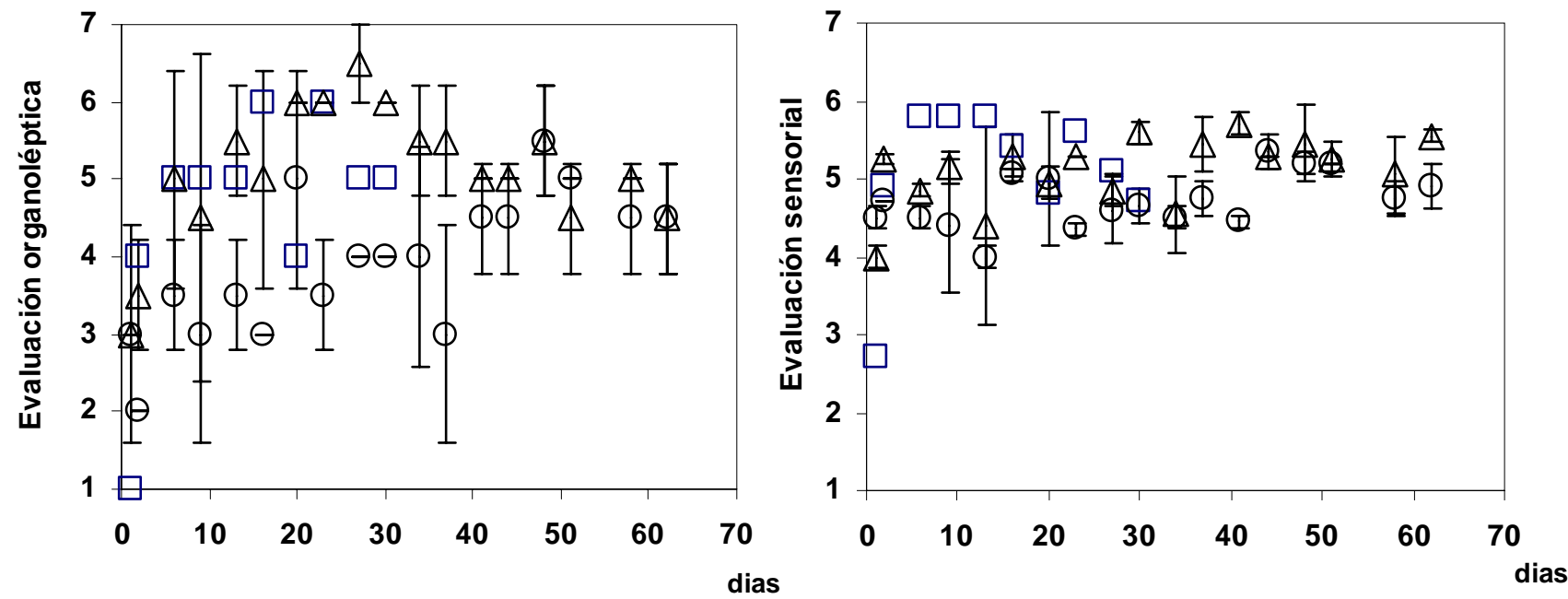

Fig. 6: Evaluación organoléptica y sensorial de color en carne cruda de vacuno envasado en MAP. $\square$ Atmósfera 0, $\bigcirc$ Atmósfera 1, $\triangle$ Atmósfera 2. Se presentan los promedios de las mediciones realizadas donde las barras de error representan $\pm \sigma$.
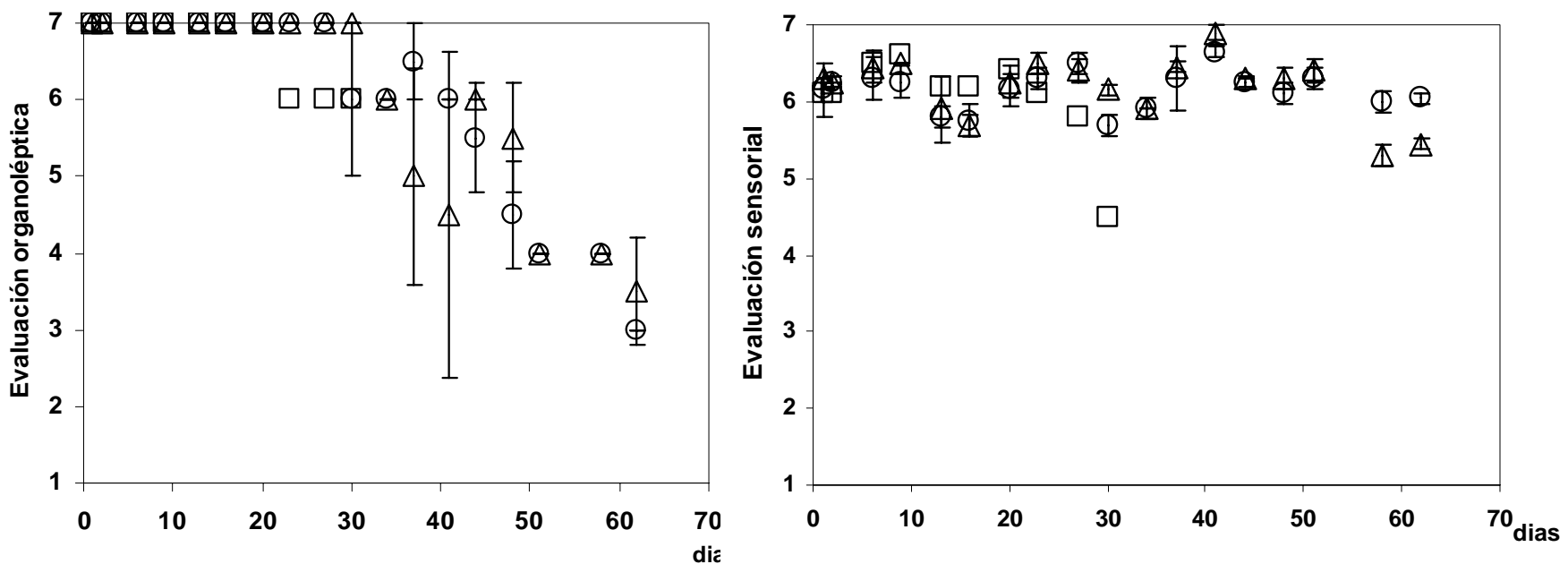

Fig. 7: Evaluación organoléptica y sensorial de olor en carne cruda de vacuno envasado en MAP.

$\square$ Atmósfera 0, $\bigcirc$ Atmósfera 1, $\triangle$ Atmósfera 2. Se presentan los promedios de las mediciones realizadas donde las barras de error representan $\pm \sigma$.

\section{Medición de color instrumental}

Mediante la medición de la coordenada colorimétrica $a^{*}$ se evaluó el efecto de las distintas atmósferas sobre la intensidad del color rojo en la carne (Fig. 8); se comparó, además, la intensidad del color para ambas caras del corte de carne: la cara expuesta al gas y la cara en contacto con la bandeja.

Se observó que la intensidad del color rojo, relacionada con el valor de $a^{*}$, para las muestras en Atmósfera 0 y 1 fue significativamente mayor $(p<0.05)$ en la cara expuesta al gas que en la en contacto con la bandeja. Sin embargo, para las muestras envasadas con mayor \%CO (Atmósfera 2) no se obtuvieron diferencias significativas entre ambas caras $(p>0.05)$. 

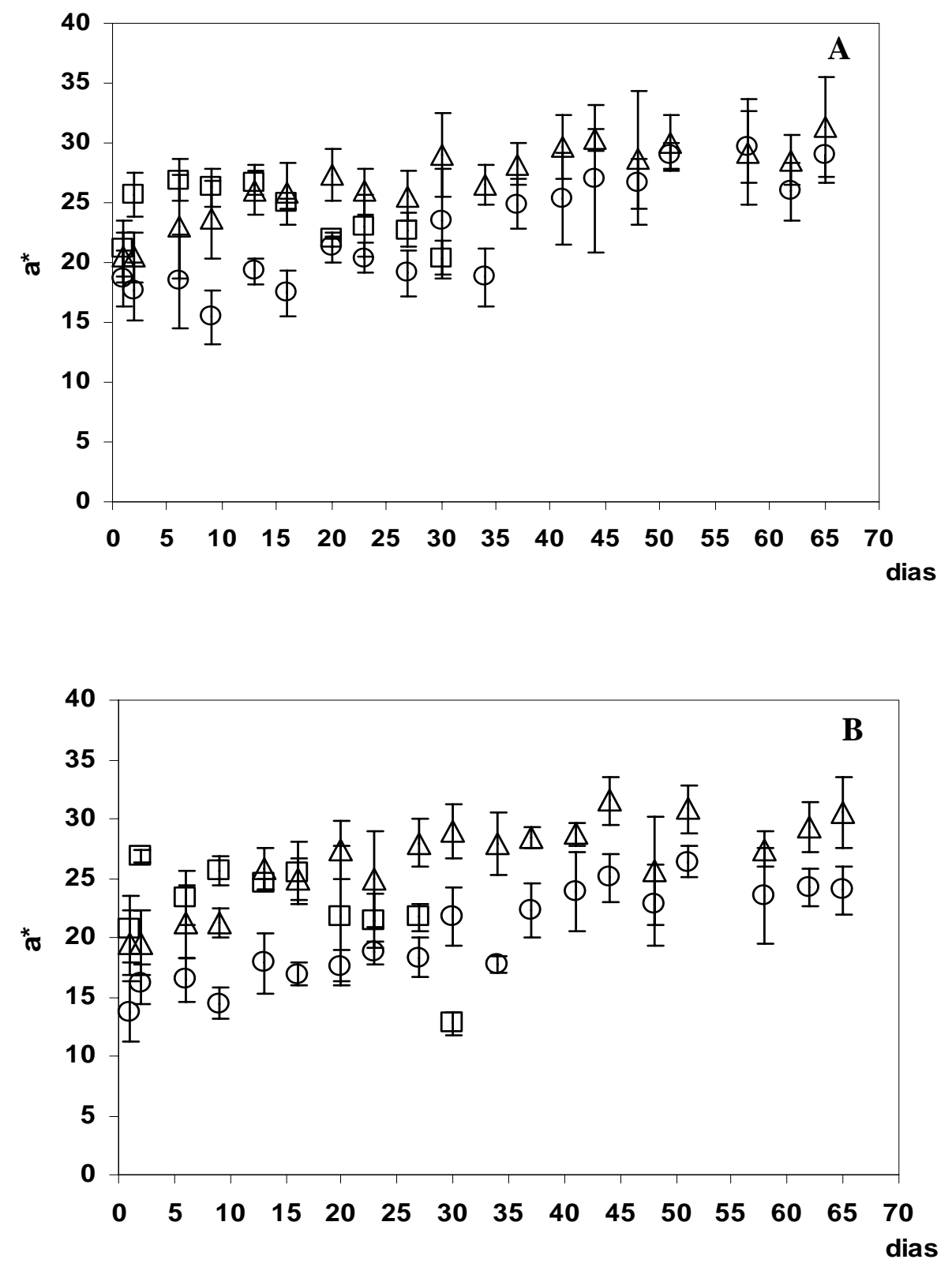

Fig. 8: Valores promedio de medición de color instrumental para carne cruda de vacuno envasado en MAP. $\square$ Atmósfera 0, O Atmósfera 1, $\triangle$ Atmósfera 2. A: $\mathbf{a}^{*}$ cara expuesta al gas, B: $a^{*}$ cara en contacto con la bandeja. Las barras de error representan $\pm \sigma$.

Durante los primeros 30 días de almacenamiento, el valor de $a^{*}$ fue significativamente superior $(p<0.05)$ para las muestras en Atmósferas 0 y 2 que para las envasadas en Atmósfera 1 (Fig. 8). Sin embargo, la intensidad del color rojo para la Atmósfera 0 presenta un valor máximo de a* a los 13 días de almacenamiento y luego disminuye, mientras que las muestras en Atmósferas con CO presentan una tendencia hacia el aumento de intensidad del rojo hasta los 50 días de almacenamiento, aproximadamente, para luego conservar sus valores de $a^{*}$ hasta el final del periodo de muestreo (65 días). Estos resultados de medición de color instrumental coinciden con los obtenidos en la evaluación organoléptica y sensorial, en donde el color mejor evaluado fue el de las muestras envasadas en Atmósfera 2; lo que demostró las ventajas del uso del CO para envasado MAP. Además, se confirma lo publicado por Sørheim (Sørheim et al., 1999), donde se establece que el envasado de carne de vacuno y cerdo en atmósfera con $0.4 \% \mathrm{CO} / 60 \% \mathrm{CO}_{2} / 40 \% \mathrm{~N}_{2}$ genera un color rojo más estable que el envasado con $70 \% \quad \mathrm{O}_{2} / 30 \% \quad \mathrm{CO}_{2}$. También, Luño et al. (2000) observaron un color más estable en carne de vacuno envasada con $\mathrm{CO} / \mathrm{CO}_{2} / \mathrm{O}_{2} / \mathrm{N}_{2}$, este fue mayor mientras mayor fue la concentración de CO utilizada. 
En resumen, los resultados demuestran las ventajas del uso de CO en atmósferas modificadas en comparación al envasado en atmósferas con alto contenido de oxígeno, para cortes de vacuno con hueso y grasa. Estas ventajas se reflejan, fundamentalmente, en una mayor vida útil de la carne, con posibles almacenamientos sobre los 60 días; menor oxidación de lípidos, lo que limita los malos olores de la carne envasada y mayor estabilidad en el color rojo de la carne, lo que induce a una mayor aceptación por parte del cliente.

Adicionalmente y basado tanto en las evaluaciones organolépticas y sensoriales como en la medición instrumental de color, es posible asegurar que una atmósfera modificada con $0.4 \% \mathrm{CO}$ es superior que la de $0.1 \%$ CO para el envasado MAP en cuanto a mayor estabilidad del color rojo de la carne.

\section{CONCLUSIONES}

El envasado de carne de vacuno con hueso y grasa en atmósferas modificadas con bajas concentraciones de $\mathrm{CO}(<0.4 \%)$ y ausencia de $\mathrm{O}_{2}$, aumenta la calidad organoléptica y sensorial del producto.

La vida útil del producto, determinada por el recuento de mesófilos aerobios, envasado en atmósfera con $\mathrm{CO}\left(0.1\right.$ y $0.4 \% \mathrm{CO}$ ) y ausencia de $\mathrm{O}_{2}$, fue de 40 a 45 días. La vida útil microbiológica de las muestras envasadas en atmósfera con alta concentración de $\mathrm{O}_{2}$ fue superior a los 30 días.

Las evaluaciones organolépticas y sensoriales indican que la intensidad de color rojo en la carne de vacuno envasada fue mayor para la atmósfera modificada con alto nivel de $\mathrm{O}_{2}$ y con $0.4 \%$ de $\mathrm{CO}$ que para las muestras envasadas en $0.1 \% \mathrm{CO}$.

No existen diferencias significativas en la medición de color instrumental y evaluación organoléptica y sensorial del color al envasar el producto en $0.4 \% \mathrm{CO} / 20 \% \mathrm{CO}_{2} / 79.6 \% \mathrm{~N}_{2}$ (Atmósfera 2 ) y $70 \% \mathrm{O}_{2}$ / $30 \mathrm{CO}_{2}$ (Atmósfera 0) durante 30 días, sin embargo, el color de la carne envasada en atmósfera con $0.4 \% \mathrm{CO}$ es más estable que el color de la carne envasada en atmósfera con $70 \% \mathrm{O}_{2}$

La utilización de atmósferas libres de $\mathrm{O}_{2}$ disminuye la oxidación de lípidos y reduce la generación de olores desagradables en la carne de vacuno.

No se observó un efecto significativo del uso de bajos niveles de $\mathrm{CO}$ sobre el exudado, $\mathrm{pH}$ y la humedad de la carne.

El uso de $\mathrm{CO}_{2}$, en presencia de $\mathrm{CO}$, retarda el crecimiento de los microorganismos aerobios y anaerobio presentes en la carne.

El aumento de la concentración de $\mathrm{CO}$ de 0.1 a $0.4 \%$ no produjo efectos significativos sobre el crecimiento bacteriano.

La utilización de 0.4\% CO/20\% $\mathrm{CO}_{2} / 79.6 \% \mathrm{~N}_{2}$ (Atmósfera 2) como atmósfera de envasado genera mejores evaluaciones en sabor y jugosidad para carne cocida que el resto de las atmósferas ensayadas (Atmósfera 1 y Atmósfera 0).

La aplicación de una atmósfera modificada con composición $0.4 \% \mathrm{CO} / 20 \% \mathrm{CO}_{2} / 79.6 \% \mathrm{~N}_{2}$, permite la conservación en buen estado de carne de vacuno con hueso y grasa, por un período de almacenamiento superior a los 40 días.

\section{AGRADECIMIENTOS}

Los autores agradecen a FONDEF (Proyecto D04i 1142) por el financiamiento de esta investigación y al estudiante memorista Raúl Avello, de Ingeniería Química, por su participación. 


\section{REFERENCIAS}

AMSA (American Meat Science Association); published in cooperation with the National Live Stock and Meat Board, Research Guidelines for Cookery, Sensory Evaluation and Instrumental Tenderness Measurements of Fresh Meat, 7-13, National Live Stock and Meat Board, Chicago, Illinois (1995).

AMSA (American Meat Science Association), The Meat evaluation Handbook (2005).

Austin, R. y otros cinco autores; Activation energy spectrum of a biomolecule: Photodissociation of carbon monoxide myoglobine at low temperature, Phys. Review Letter: 32 (8) 403-405 (1974).

Blomberg, L., M. Blomberg, y A. Siegbahn; Theorical study on the binding of $\mathrm{O}_{2}, \mathrm{NO}$ and $\mathrm{CO}$ to heme proteins, J. Inorganic Biochem.: 99, 949-958 (2005).

Brannan, G y E. Decker; Peroxynitrite-Induced Oxidation of Lipids: Implications for Muscle Foods, J. Agric. Food Chem.: 49, 3074-3079 (2001).

Brody, L.A.; The case for -or againts- case-ready fresh red meat in the United Stated. Food Technology: 54(8), 153-156 (2000)

Campo, M. y otros cinco autores; Flavour perception of oxidation in beef, Meat Science: 72 303-311 (2006).

Chow, C.K. (editor); Fatty acid in foods and their health implications, Publisher: Marcel Dekker, New York (1992).

Dixon, Z. y otros seis autores; Effect of degree of sanitation from slaughter through fabrication on the microbiological and sensory characteristics of beef, J. Food Protection: 54 (20) 200-207 (1991).

Farber, J. y L. Dodds; Principles of modified atmosphere and sous vide product packaging, Technomic Publishing Company, Inc. (1995).

FDA, U. S. Food and Drug Administration, Bacteriological Analytical Manual (BAM) Online (2001), http://www.cfsan.fda.gov/ ebam/bam-toc.html. Acceso: 10 de enero de (2008).

Gill, C.; Extending the storage life of raw chilled meats, Meat Science: 43 (1) 99-109 (1996).

Hui, Y., I. Guerrero y M. Rosmini; Ciencia y Tecnología de Carnes, Ed. Limusa, México (2006).

Jayasingh, P., D.P. Cornforth, C.E. Carpenter y D. Whittier; Evaluation of carbon monoxide treatment in modified atmosphere packaging or vacuum packaging to increase color stability of fresh beef, Meat Science: 59, 317-324 (2001)

John, L. y otros cinco autores; Color an thiobarbituric acid values of cooked top sirloin steaks packaged in modified atmospheres of $80 \%$ oxygen, or $0.4 \%$ carbon monoxide, or vacuum, Meat Science: 69, 441-449 (2005).

Luño, M., P. Roncalés, D. Djenane, y J. Beltrán; Beef shelf life in low $\mathrm{O}_{2}$ and high $\mathrm{CO}_{2}$ atmospheres containing different low CO concentrations, Meat Science: 55, 413-419 (2000).

Masana, M., L. Meichtri y R. Rodríguez; "Determinación de la vida útil en cortes de bovinos. Mayor Calidad por más tiempo. Instituto Tecnológico de alimentos". INTA, Cautelar (2006). www.inta.gov.ar/ediciones/idia/carne/carnef03.pdf. Acceso: 15 de diciembre de (2007).

Méndez, D., P. Recio, B. Béjar y C. Fernández; "Coordenadas del color" (2006), http://campusvirtual.uma.es/tdi/alumnos/tdi02/, Acceso: 2 de diciembre de (2007). 
Nissen, H., O. Sørheim y R. Dainty; Effects of vacuum, modified atmospheres and storage temperature on the microbial flora of packaged beef, Food Microbiology: 13, 183-191 (1996).

Otwell, W.S., H.G. Kristinsson, M.O. Balaban; Modified Atmospheric processing and packaging of fish, First Edition, 103-115, Blackwell Publishing, IOWA, USA (2006).

Pensel, N.; Influence of experimental conditions on porcine muscle and its effect on oxidation, M. Sc. Thesis, Dept. of Animal Sciences, The Ohio State University, Columbus, Ohio, USA (1990). http://ohioline.osu.edu/sc172/sc172 6.html. Acceso: 14 de septiembre de (2007).

SCF, Scientific Committee on Food; Opinion of the scientific committee on food on the use of carbon monoxide as component of packaging gases in modified atmosphere packaging for fresh meat, European Commission (adopted on 13 december 2001), Health and Consumer Protection Directorate General, http://ec.europa.eu/food/fs/sc/scf/out112 en.pdf. Acceso: 15 de octubre de (2007).

Sørheim, O., T. Aune y T. Nesbakken; Technological hygienic and toxicological aspects of carbon monoxide used in modified atmosphere packaging of meat, Trends in Food Science and Technology: 8, 307, 312 (1997).

Sørheim, O., H. Nissen y T. Nesbakken; The storage life of beef and pork packaged in a modified atmosphere with low carbon monoxide and high carbon dioxide, Meat Sci.: 52, 157-164 (1999).

Zhang, M., y S. Sundar; Effect of Oxygen Concentratio on the Shelf-Life of Fresh Pork Packed in Modified Atmosphere, Packaging Technology and Science: 18, 217-222 (2005). 
